\title{
Coherence properties of modeless lasers
}

\author{
Hugues Guillet de Chatellus \\ Laboratoire de Spectrométrie Physique \\ UMR 5588 CNRS-Université Joseph Fourier \\ 140 Av. de la Physique, \\ BP 87 - 38402 Saint Martin d'Hères \\ France \\ E-mail: hguillet@spectro.ujf-grenoble.fr
}

\section{Jean-Paul Pique}

Laboratoire de Spectrométrie Physique

UMR 5588 CNRS-Université Joseph Fourier

140 Av. de la Physique,

BP 87 - 38402 Saint Martin d'Hères

France

E-mail: pique@spectro.ujf-grenoble.fr

\begin{abstract}
Most of classical light sources show a close similarity between their first and second order correlation functions (resp. $\mathrm{g}^{(1)}$ and $\mathrm{g}^{(2)}$ ) functions. We present here the original coherence properties of a peculiar type of laser named modeless laser or Frequency Shifted Feedback (FSF) laser where the $\mathrm{g}^{(1)}$ and $\mathrm{g}^{(2)}$ functions show a different behaviour. We calculate and evidence experimentally the first and second order correlation functions of modeless lasers, through measurements of the homodyne beat signal and interferometric autocorrelation of a dye FSF laser at the output of a Michelson interferometer. Whereas the degree of first-order coherence vanishes beyond the coherence length of the FSF source, the degree of second-order coherence exhibits periodic revivals far beyond the coherence length, with a period equal to the cavity roundtrip time. Our observations are in good agreement with the theoretical treatment of Yatsenko et al. (Opt. Comm. 282 (2009) 300) [1].
\end{abstract}

Quantum of Quasars workshop

Grenoble, France

December 2-4, 2009 


\section{Introduction: field and intensity temporal correlations}

Coherence properties of light fields are of primary importance both from a theoretical and and experimental perspective in optics. The field temporal correlations - or degree of first order coherence- is linked to the phase properties of a light field whereas the intensity temporal correlation (or degree of second order coherence) is linked its amplitude properties. Classical imaging uses the degree of first order coherence but because of the strong influence of usual propagation media on the optical phase, resulting in the degradation of the optical wavefront in inhomogeneous media (atmosphere, water, biological tissues...), it could be highly interesting to perform imaging by using instead the degree of second order coherence. The famous HanburyBrown Twiss (HBT) stellar interferometer opened a new way of thinking coherence in optics and at the same time demonstrated new possibilities of imaging, that are currently investigated through the field of quantum imaging [2]. In the HBT experiment, the astronomers measure the angular diameter of bright stars by measuring the degree of second order coherence, and make the hypothesis that the degree of second order coherence is directly linked to the degree of first order coherence [3]. In fact most of usual light sources show this property: thermal sources, chaotic sources, single mode or multimode lasers. But here we show that this is not always the case and we study an original laser source, called modeless laser of FSF laser (for frequency shifted feedback), which shows decorrelated $\mathrm{g}^{(1)}$ and $\mathrm{g}^{(2)}$ functions. The $\mathrm{g}^{(1)}$ function of modeless lasers is limited to the domain of coherence while the $\mathrm{g}^{(2)}$ exhibits periodic revivals, far beyond the (first order) coherence time of the laser. We therefore demonstrate that modeless laser shows time delays for which the fields are mutually incoherent but the amplitudes are coherent.

\subsection{Definitions}

We restrict our study to classical light fields described by continuous functions of time, instead of quantum operators. Recall that the degree of first order coherence (or field temporal correlation function) is defined by [4]:

$$
g^{(1)}(\tau)=\frac{\left\langle E(t) E^{*}(t+\tau)\right\rangle}{\left\langle E(t) E^{*}(t)\right\rangle}
$$

where $\tau$ is the time delay, $E(t)$ is the complex electrical light field and the brackets denote ensemble averages. We implicitely assume a stationnary process, that is $\mathrm{g}^{(1)}$ is independent from t. Defining the instantaneous intensity as $I(t)=E(t) E^{*}(t)$, we define similarly the degree of second order coherence (or intensity temporal correlation function) by:

$$
g^{(2)}(\tau)=\frac{\langle I(t) I(t+\tau)\rangle}{\langle I(t)\rangle^{2}}=\frac{\left\langle E(t) E^{*}(t) E(t+\tau) E^{*}(t+\tau)\right\rangle}{\left\langle E(t) E^{*}(t)\right\rangle^{2}}
$$




\subsection{Properties}

We consider a light field in the form

$$
E(t)=E_{0}(t) e^{i \omega_{0} t+i \varphi(t)}
$$

where $E_{0}(t)$ and $\varphi(t)$ correspond respectively to amplitude and phase fluctuations. It can easily been shown that $\mathrm{g}^{(1)}$ depends on both phase and amplitude whereas $\mathrm{g}^{(2)}$ depends only on amplitude fluctuations. It is also instructive to calculate the Fourier transform of $\mathrm{g}^{(1)}$ and $\mathbf{g}^{(2)}$.

In the case of $\mathrm{g}^{(1)}$,

$$
\begin{aligned}
\int g^{(1)}(\tau) e^{-i \omega \tau} d \tau & =\int\langle E(t) E(t+\tau)\rangle e^{-i \omega \tau} d \tau \\
& =\left|\int E(t) e^{-i \omega t} d t\right|^{2} \\
& =S_{o p t}(\omega)
\end{aligned}
$$

The Fourier transform of $\mathrm{g}^{(1)}$ is equal to the optical spectrum, provided the WienerKhinchine theorem can be applied, which is the case for stationary processes.

In the case of $\mathrm{g}^{(2)}$ :

$$
\begin{aligned}
\int g^{(2)}(\tau) e^{-i \Omega \tau} d \tau & =\int\langle I(t) I(t+\tau)\rangle e^{-i \Omega \tau} d \tau \\
& =\left|\int I(t) e^{-i \Omega t} d t\right|^{2} \\
& =S_{R F}(\Omega)
\end{aligned}
$$

Provided the Wiener-Khinchine theorem can be applied, the Fourier transform of $g^{(2)}$ is equal to the power spectrum of the time fluctuations of the photocurrent, called radio frequency spectrum. Therefore both $\mathrm{g}^{(1)}$ and $\mathrm{g}^{(2)}$ functions can be measured indirectly through their Fourier conjugate.

\section{Coherence properties of usual classical light sources}

In this paragraph we review the first and second order coherence properties of classical usual light sources, to illustrate the fact that in most of cases, both the degrees of first and second order coherence show a large degree of similarity.

\subsection{Discrete emitters}

\subsubsection{Single emitter}

We consider here the case of a single light emitter, whose emission process is randomly dephased by collision. This is a case of a single atom emitter in a gas discharge lamp. We also assume that the lifetime duration of the excited level is long compared to the optical period to neglect the variation of the amplitude of the emitted wave. The electric field can be written as:

$$
E(t)=E_{0} e^{i\left(\omega_{0} t+\varphi(t)\right)}
$$


In that case the $\mathrm{g}^{(1)}$ function is localized around zero with a width (coherence time) linked to the average time between successive collisions. The $\mathrm{g}^{(2)}$ function is equal to 1 for all $t$. In the case of a single emitter, the $g^{(1)}$ and $g^{(2)}$ functions are not similar.
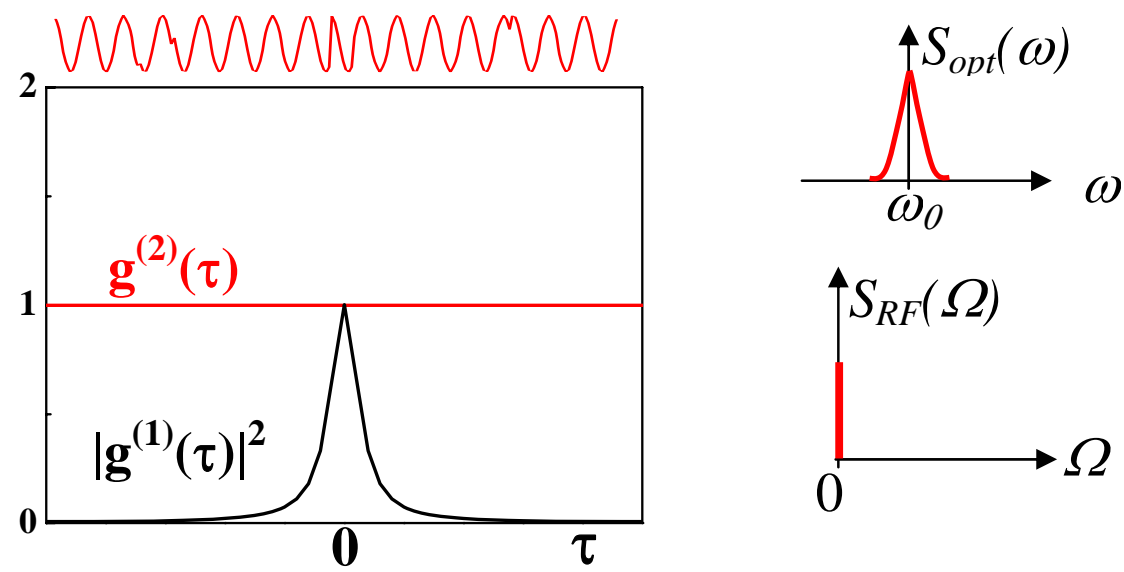

Figure 1. Top left: electric field sequence emitted by a single atom submitted to dephasing collisions. Bottom left: theoretical $\mathrm{g}^{(1)}$ and $\mathrm{g}^{(2)}$ functions. Right: optical (top) and RF spectrum (bottom).

\subsubsection{N emitters}

We now turn to the case of $\mathrm{N}$ independent emitters. The electric field can be expressed as:

$$
E(t)=E_{0} e^{i \omega_{0} t} \sum_{n=1}^{N} e^{i \varphi_{n}(t)}
$$

The $\mathrm{g}^{(2)}$ function can be calculated and after averaging out the different interference terms and one gets:

$$
g^{(2)}(\tau)=1+\frac{N-1}{N}\left|g^{(1)}(\tau)\right|^{2}
$$
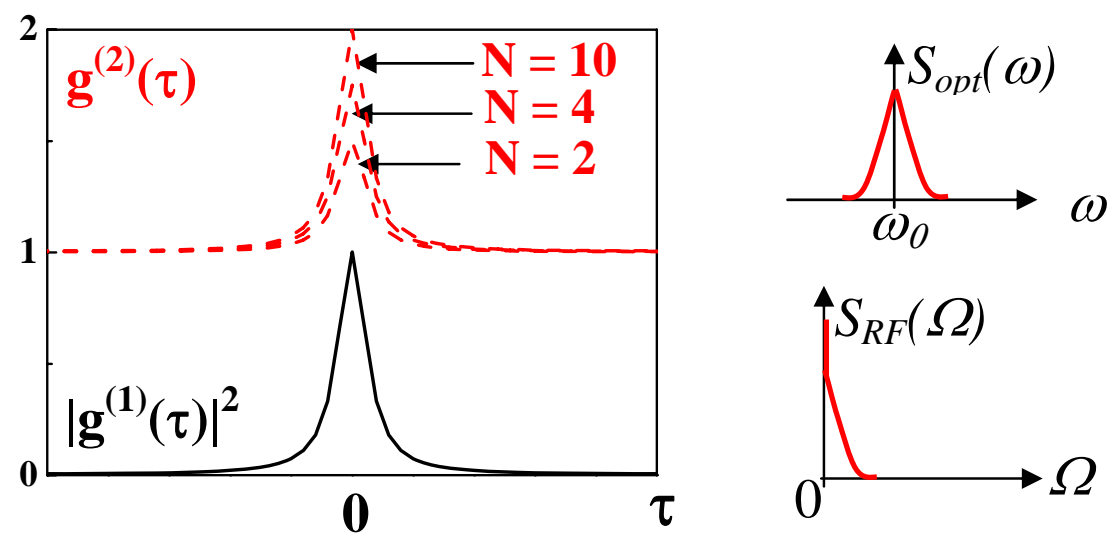

Figure 2. Left: theoretical $\mathrm{g}^{(1)}$ and $\mathrm{g}^{(2)}$ functions of multiple independent emitters. Right: optical (top) and RF spectrum (bottom). 


\subsubsection{Chaotic light sources}

We now turn to the case of a continuous distribution of emitters. Then the electric field can be described as a random process (chaotic). If one assumes gaussian statistics for $E(t)$ the following factorization rule holds:

$\left\langle E(t) E^{*}(t) E(t+\tau) E^{*}(t+\tau)\right\rangle=\left\langle E(t) E^{*}(t)\right\rangle\left\langle E(t+\tau) E^{*}(t+\tau)\right\rangle+\left\langle E(t) E^{*}(t+\tau)\right\rangle\left\langle E(t+\tau) E^{*}(t)\right\rangle$

Replacing this expression into the deginition of $\mathrm{g}^{(2)}$ leads to the general relation:

$$
g^{(2)}(\tau)=1+\left|g^{(1)}(\tau)\right|^{2}
$$

The $\mathrm{g}^{(2)}$ function is therefore closely related to the $\mathrm{g}^{(1)}$ function for a large variety of light sources: incandescent lamp, gas discharge lamp, thermal cavity, spontaneous emission, amplified spontaneous emission... This relation is at the basis of the HBT stellar interferometer for which the coherence length of intensity fluctuations has been identified to the coherence length of the electric field to deduce the diameter of the star. For a null time delay, the $\mathrm{g}^{(2)}$ is equal to two. In term of photon statistics this relation is the signature of photon bunching. In terms of intensity fluctuations, the variance of the process obeys the relation:

$$
\sigma^{2}=\left\langle I(t)^{2}\right\rangle-\langle I(t)\rangle^{2}=\langle I(t)\rangle^{2}
$$

which means that the intensity fluctuations of a chaotic light source are comparable to the mean intensity.

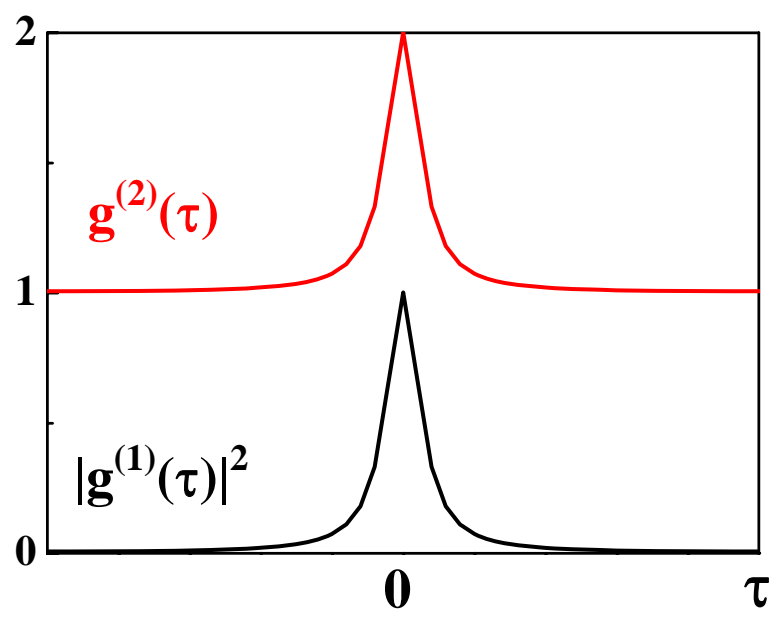

Figure 3. $\mathrm{g}^{(1)}$ and $\mathrm{g}^{(2)}$ functions of chaotic light sources. 


\subsection{Laser sources}

\subsubsection{Single mode laser}

The case of a single mode laser is particularly simple. The amplitude of the electric field is stabilized by the gain. The electric field can be written as

$$
E(t)=E_{0} e^{i \omega_{0} t}
$$

and the related $g^{(1)}$ and $g^{(2)}$ functions are equal to 1 , for any value of the time delay. The corresponding optical and RF spectrum are Dirac functions.
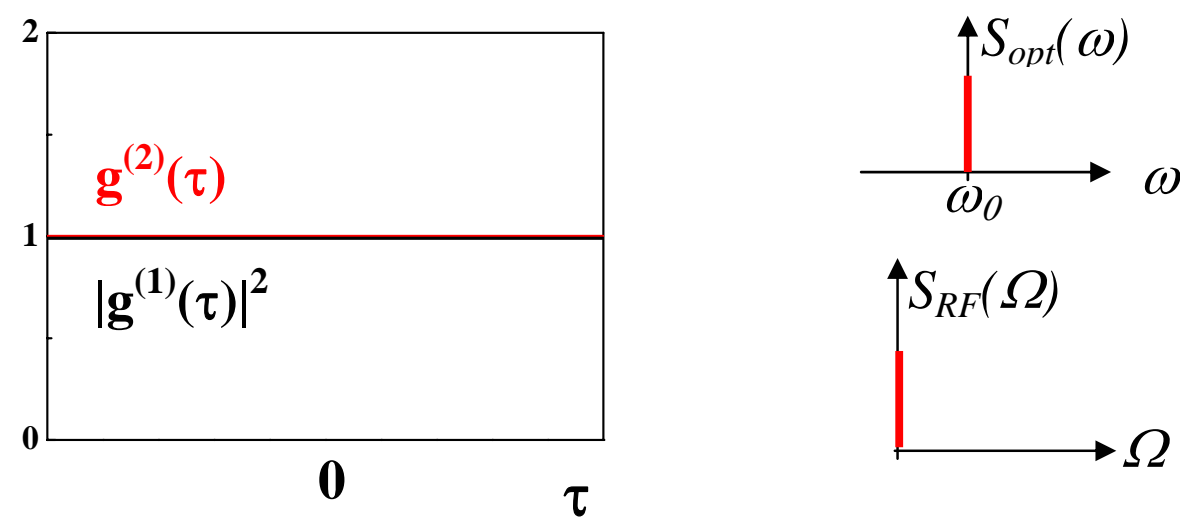

Figure 4. Left: theoretical $\mathrm{g}^{(1)}$ and $\mathrm{g}^{(2)}$ functions of a single mode laser. Right: optical (top) and RF spectrum (bottom).

\subsubsection{Multimode laser}

We treat the case of a multimode laser with no specific phase relationship between the modes. Phases are assumed random and we neglect the competition arising in the laser gain medium and the related dynamic effects. We assume and electric field in the form:

$$
E(t)=E_{0} e^{i \omega_{0} t} \sum_{n=-N}^{N} \zeta(n) e^{i n(\delta \omega) t} e^{i \varphi_{n}(t)}
$$

where $\omega_{0}$ is the average angular frequency, $\zeta(n)$ is the amplitude of the ${ }^{\text {th }}$ mode, $\delta \omega$ is the laser free spectral range and $\varphi_{n}$ is the phase of the $\mathrm{n}^{\text {th }}$ mode. In that case, the $\mathrm{g}^{(1)}$ and $\mathrm{g}^{(2)}$ functions show periodic revivals at integer multiples of the cavity roundtrip time ( $\tau_{r}=2 \pi / \delta \omega$ ). The temporal width of the peaks is the coherence time of the laser, whereas the amplitude of the peaks decay as a function of the coherence time of the phase $\varphi_{n}$. The $\mathrm{g}^{(2)}$ function at zero delay is equal to 2 (photon bunching). 

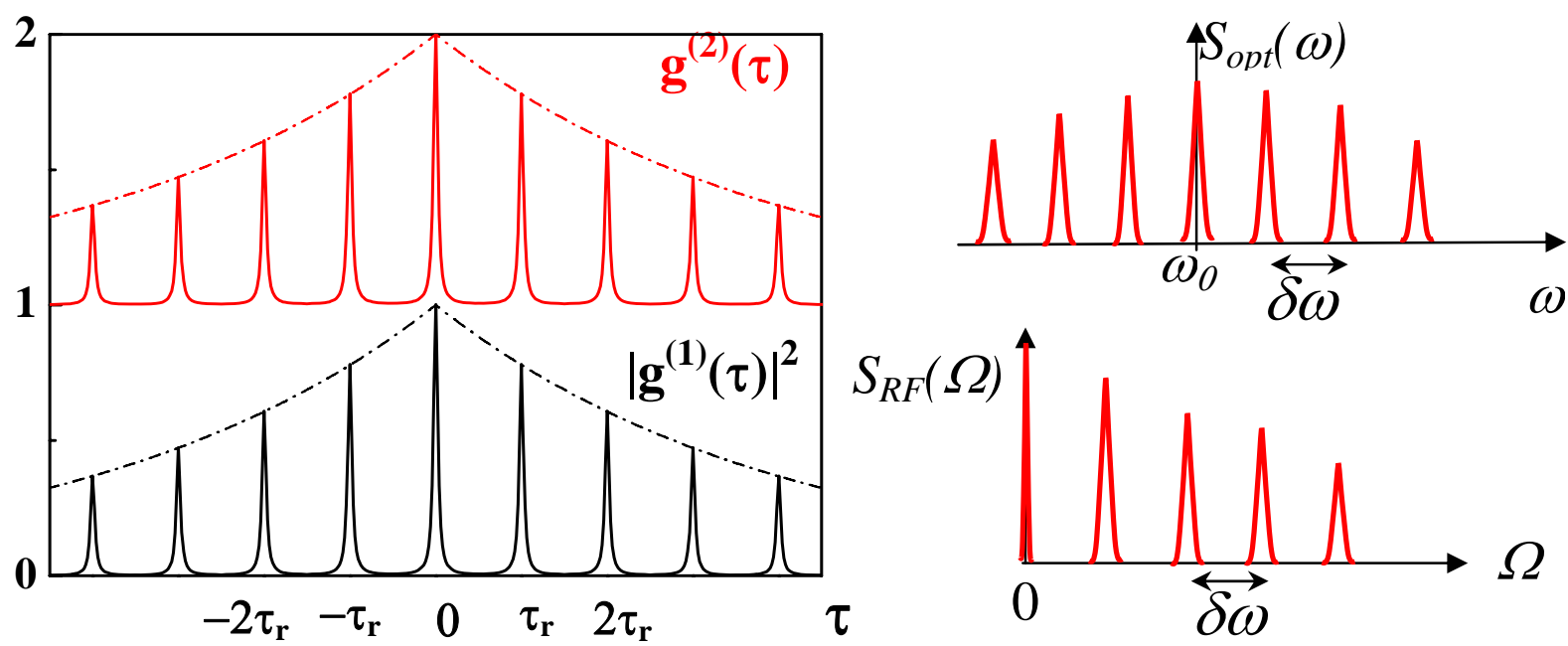

Figure 5. Left: theoretical $\mathrm{g}^{(1)}$ and $\mathrm{g}^{(2)}$ functions of a multimode laser with a large number of modes. Right: optical (top) and RF spectrum (bottom).

In the case where the phases of the modes have a definite relationship as in the case of mode-locked lasers, the output light field can be pulsed. In that case, the $\mathrm{g}^{(1)}$ function remains the same but the $\mathrm{g}^{(2)}$ function changes: the height of the peaks of the $\mathrm{g}^{(2)}$ function increase and can reach very large values whereas the background between two peaks falls down to zero.

\section{Theoretical coherence properties of modeless laser}

It appears from the review of the $g^{(1)}$ and $g^{(2)}$ functions of usual classical sources that there is a strong similarity between the $\mathrm{g}^{(1)}$ and the $\mathrm{g}^{(2)}$ function of a classical light source. But this is not always the case: in the following we focus on the coherence properties of a particular laser source called modeless laser and we show both theoretically and experimentally that the $\mathrm{g}^{(1)}$ and $\mathrm{g}^{(2)}$ functions are decorrelated.

\subsection{The passive cavity model}

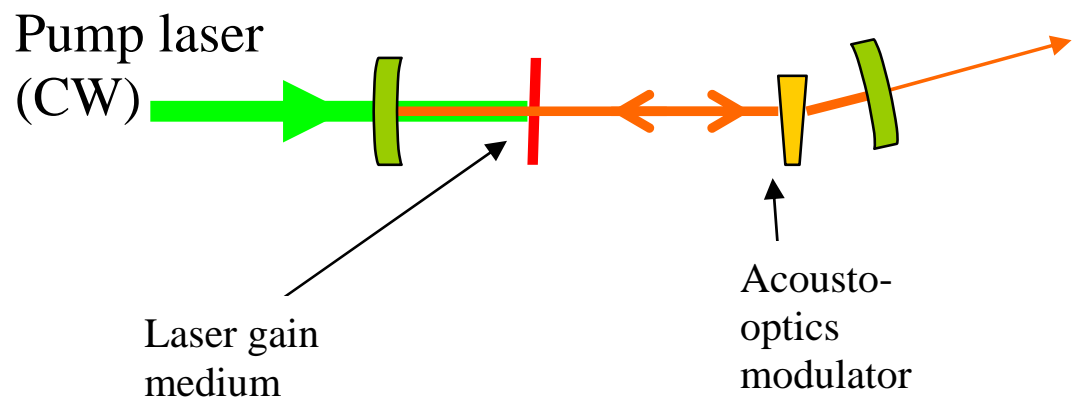




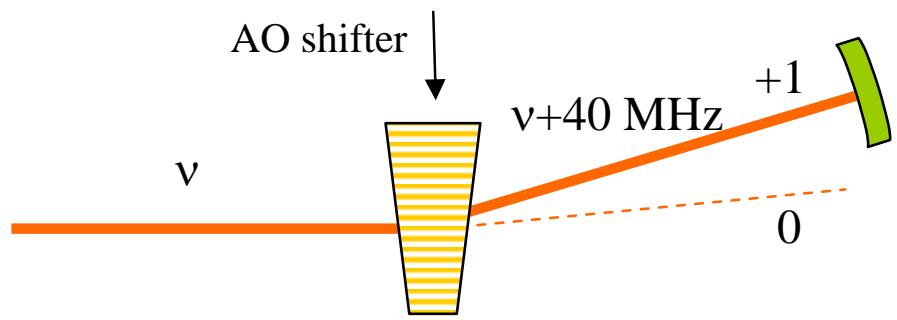

Figure 6. Top: sketch of a modeless laser. Bottom: principle of operation of the acousto-optics modulator. The acoustic frequency is $40 \mathrm{MHz}$.

A modeless (or FSF for Frequency Shifted Feedback laser) is a laser cavity in which a frequency shifter has been inserted [5]. This shifter is most of the time an acousto-optics (AO) modulator where an acoustic grating is created in a crystal and diffracts the photons while increasing their frequency by the frequency of the acoustic modulation. The laser cavity is closed on the +1 order of the diffraction grating. Therefore a photon experiences a frequency shift $\Delta$ per roundtrip equal to twice the AO frequency.

To express the output electric field we consider the model of the passive cavity: we neglect the spectral dependence of the gain. Therefore the cavity can be described as a regenerative cavity, seeded by the field resulting from the spontaneous emission of the gain medium and shifted in frequency at each roundtrip [1].

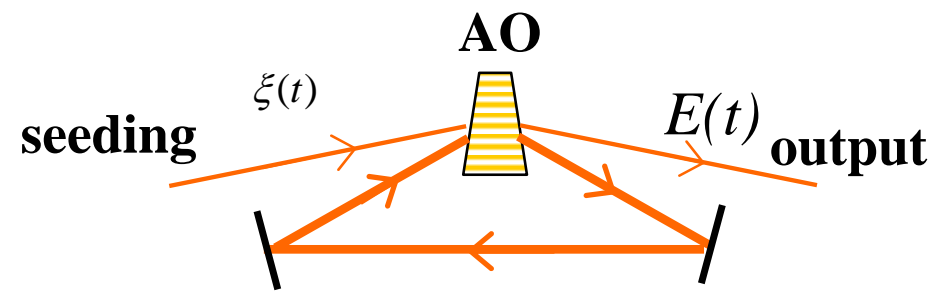

Figure 7. Principle of the passive cavity model.

This seeding field $\xi(t)$ can be defined equivalently by its temporal correlation function of by its spectrum.

$$
\begin{aligned}
& F(\tau)=\left\langle\xi(t) \xi^{*}(t+\tau)\right\rangle=I_{0} e^{-i \omega_{0} \tau-\gamma|\tau|} \\
& I(\omega)=\frac{\gamma / \pi}{\left(\omega-\omega_{0}\right)^{2}+\gamma^{2}} I_{0}
\end{aligned}
$$

The electric field at the output of the laser can be defined by the recurrence relation:

$$
E(t)=\xi(t)+R E\left(t-\tau_{r}\right) e^{-i \Delta t}
$$

where $R$ is the diffraction efficiency of the AOM ( $R$ is close to 1 ).

In first approximation,

$$
I(t) \simeq I\left(t-\tau_{r}\right)
$$

which shows the periodic nature of the light intensity of the FSF laser. This periodicity is therefore present in the $\mathrm{g}^{(2)}$ function [6]. This property is not specific to modeless 
lasers and is also shared by multimode lasers. On the contrary, in the case of a modeless laser the electric field can be approximated by:

$$
E(t) \simeq E\left(t-\tau_{r}\right) e^{-i \Delta t}
$$

which shows that the electric field of a modeless laser is intrinsically chirped.

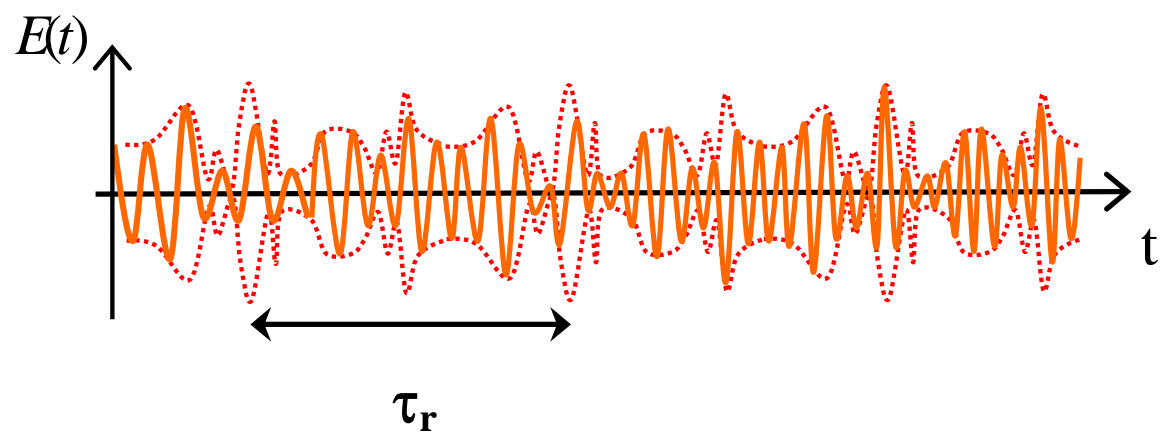

Figure 8. Intuitive picture of the output field of a modeless laser, showing repetitive amplitude fluctuations with an intrinsic chirp in the instantaneous frequency.

\section{$3.2 \mathrm{~g}^{(1)}$ and $\mathrm{g}^{(2)}$ functions}

By using the correlation function of the $\xi$ field, one can calculate analytically the $\mathrm{g}^{(1)}$ and $\mathrm{g}^{(2)}$ functions of the modeless laser and one obtains respectively [6]:

$$
\overline{g^{(1)}(\tau)}=e^{-i \omega_{0} \tau-\gamma|\tau|} \quad g^{(2)}(\tau)=1+\frac{\sum_{p=0}^{\infty} R^{2 p} \sum_{n=0}^{\infty}\left|F\left(\tau-(p-n) \tau_{r}\right)\right|^{2}}{I_{0}^{2} \sum_{p=0}^{\infty} R^{2 p}}
$$

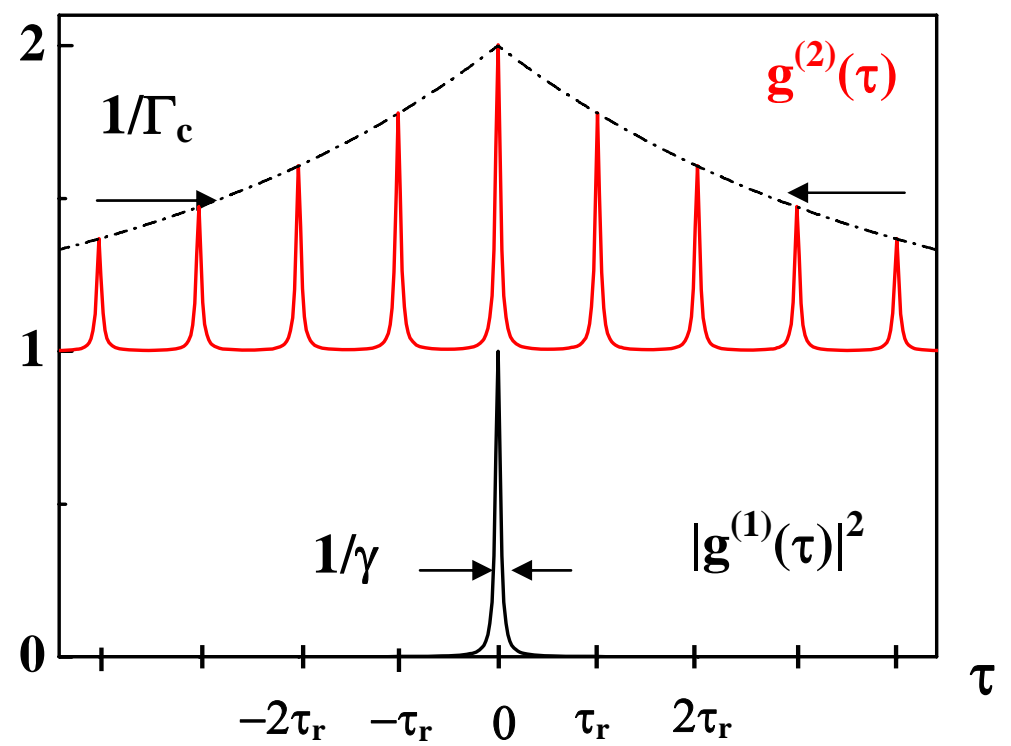

Figure 9. Theoretical $\mathrm{g}^{(1)}$ and $\mathrm{g}^{(2)}$ functions of a modeless laser 
Therefore the modeless laser exhibits decorrelated $\mathrm{g}^{(1)}$ and $\mathrm{g}^{(2)}$ functions. The $\mathrm{g}^{(1)}$ function is localized within the coherence time of the laser while the $g^{(2)}$ function exhibits periodic revivals, with a period equal to the cavity roundtrip time.

\section{Experimental measurement of the degree of first and second order coherence of modeless lasers}

We turn to the experimental determination of the $\mathrm{g}^{(1)}$ and $\mathrm{g}^{(2)}$ functions of a dye modeless laser operating at $589 \mathrm{~nm}$. The spectral width is equal to $85 \mathrm{GHz}$. The $\mathrm{g}^{(1)}$ function is measured in a conventional manner by scanning an interferometer. Concerning the $\mathrm{g}^{(2)}$ function, because of the short coherence time of our modeless laser (about $10 \mathrm{ps)}$ we chose to perform second harmonic generation (SHG) at the output of the interferometer to measure the autocorrelation trace of the modeless laser, in the same way as the measurement of ultrashort pulses. We used a long interferometer where the path difference between both arms can exceed one cavity length, enabling to reach delays larger than the cavity roundtrip time $\tau_{\mathrm{r}}[6]$.

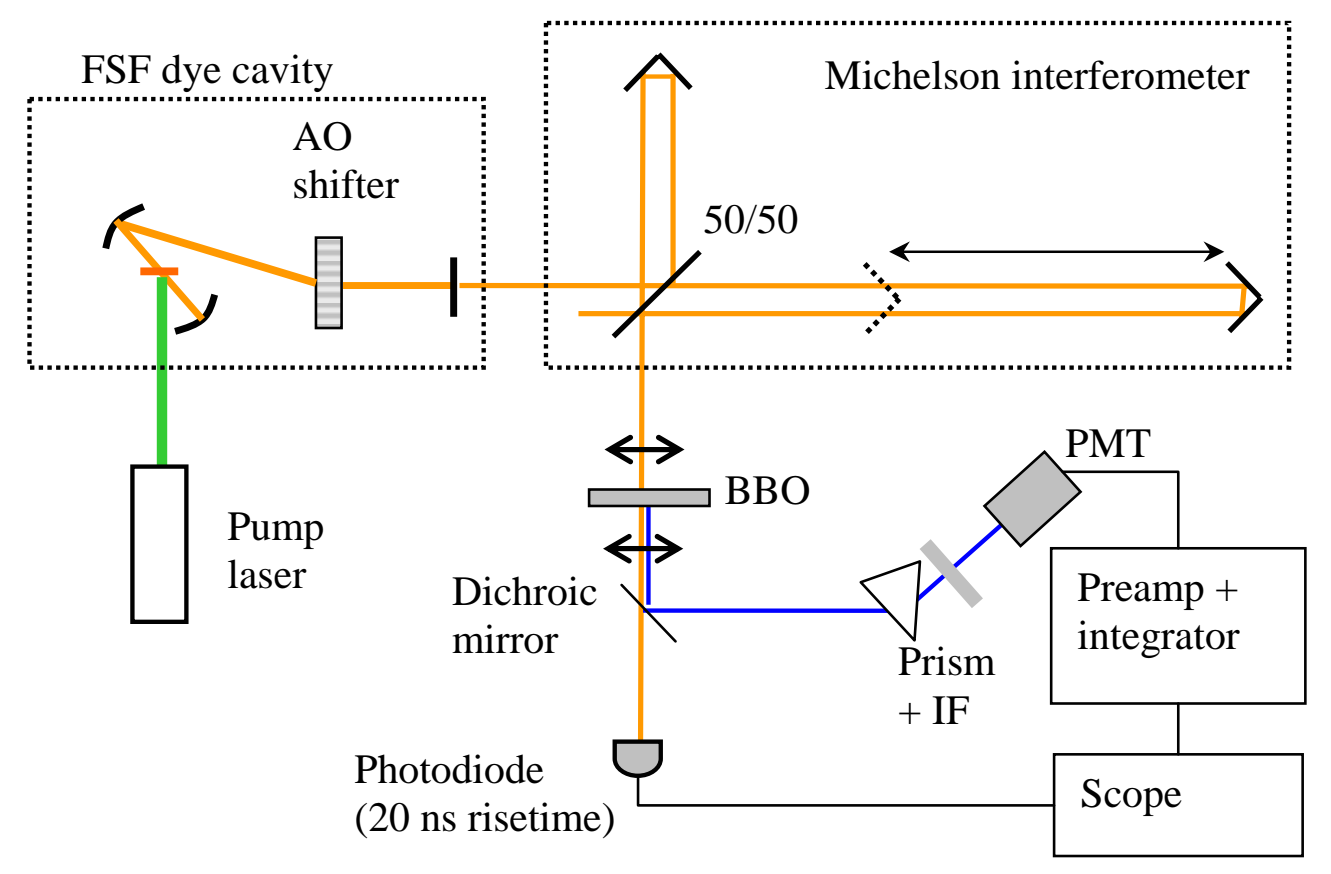

Figure 10. Experimental setup for the measurement of the $\mathrm{g}^{(1)}$ and $\mathrm{g}^{(2)}$ functions of the modeless laser. The $\mathrm{g}^{(2)}$ is deduced from the collinear autocorrelation function measured by the frequency doubling in the nonlinear crystal at the output of the interferometer.

The theoretical autocorrelation trace can be calculated using the expression of the modeless laser field. 


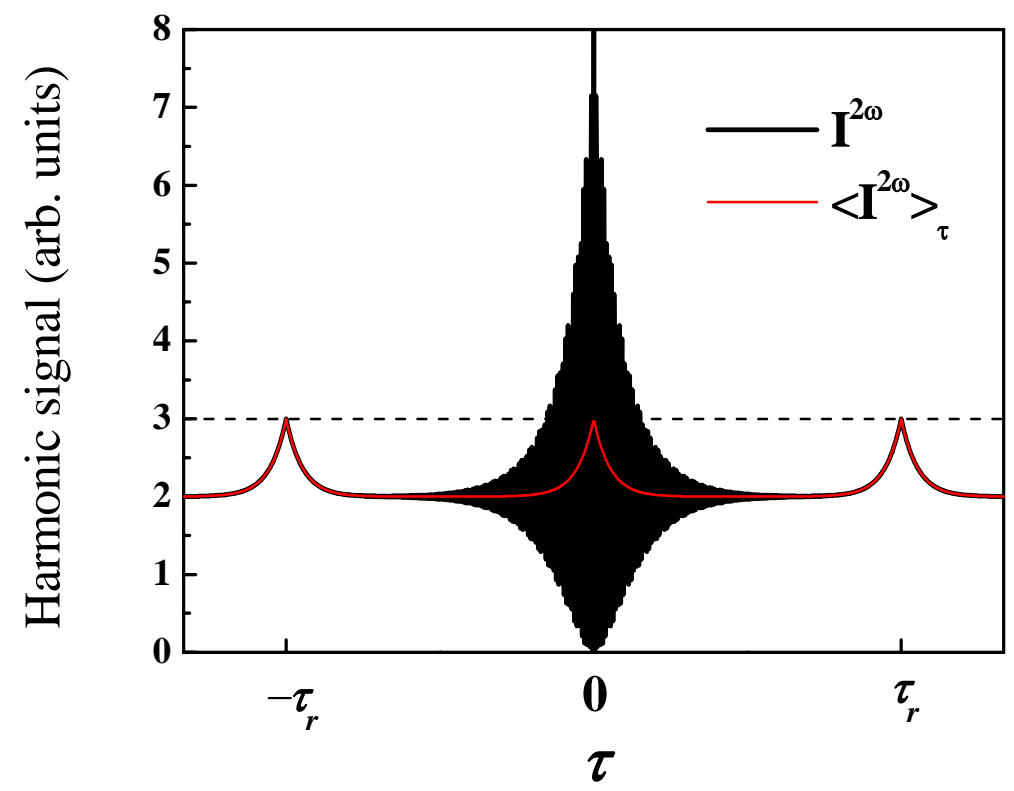

Figure 11. Theoretical autocorrelation trace of the field of the modeless laser. Black: no averaging is performed over the fringes. Red: fringes are averaged out. Note the ratio 3:2 of the height of the peak to the background.

The experimental results show that the $\mathrm{g}^{(1)}$ function is localized within the coherence length and vanishes elsewhere. On the contrary the autocorrelation trace exhibits a peak when the time delay is equal to the cavity roundtrip time. The measured ratio of the height of the peak to the background is close to 3:2, which is in good agreement with the theoretical prediction from the passive cavity model.
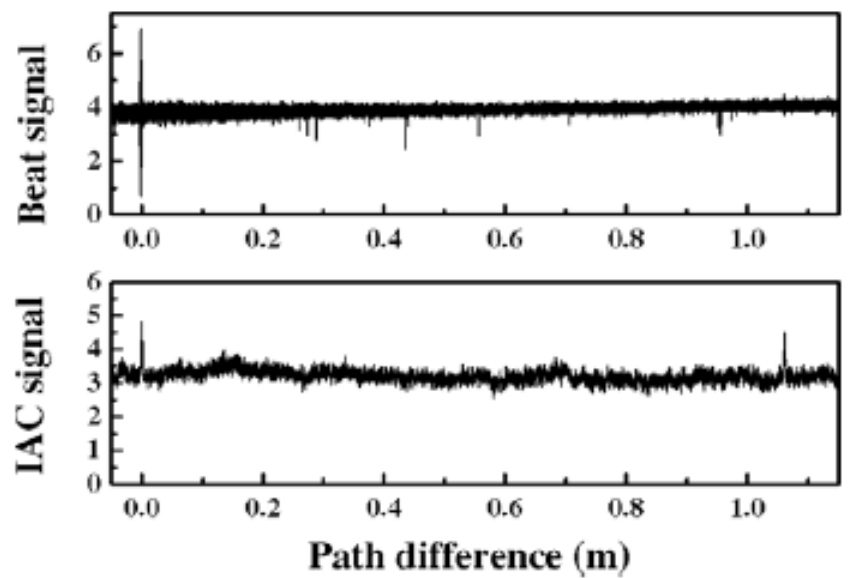

Figure 12. Experimental results. Top: interferogram of the modeless laser. The fringes are localized at null path difference. Bottom: Interferometric autocorrelation trace. Two peaks are localized: one at zero path delay, and the second one at a path delay equal to $1.06 \mathrm{~m}$ (the length of a cavity roundtrip). 
In conclusion, we have shown that contrary to most of usual light sources, the $g^{(1)}$ and $g^{(2)}$ functions of a modeless (or FSF) laser show a different bahaviour. In other words, the modeless laser exhibits intensity correlation where the field is no more (first order) coherent Possible applications concern quantum imaging [2]. This original behaviour is to be related to the very specific radio-frequency properties of modeless lasers [1,6].

\section{References}

[1] L. P. Yatsenko, B. W. Shore, K. Bergmann, “Coherence in the output of frequency shifted feedback lasers”, Opt. Comm. 282, 300 (2009)

[2] Y. Shih, “Quantum Imaging”, IEEE J. Sel. Top. Quant. Elec. 13, 1016 (2007)

[3] R. Hanburry Brown, R. Q. Twiss, “Correlation between photons in two coherent beams of light”, Nature 177, 27 (1956)

[4] R. Loudon, “The Quantum Theory of Light”, third ed. Oxford University Press, New York 2000

[5] F. V. Kowalski, P. D. Hale, S. J. Shattil, „Broadband continuous-wave laser”, Opt. Lett. 13, 822 (1988)

[6] H. Guillet de Chatellus, J.-P. Pique, « Statistical properties of frequency shifted feedback lasers», Opt. Comm. 283, 71 (2010) 